
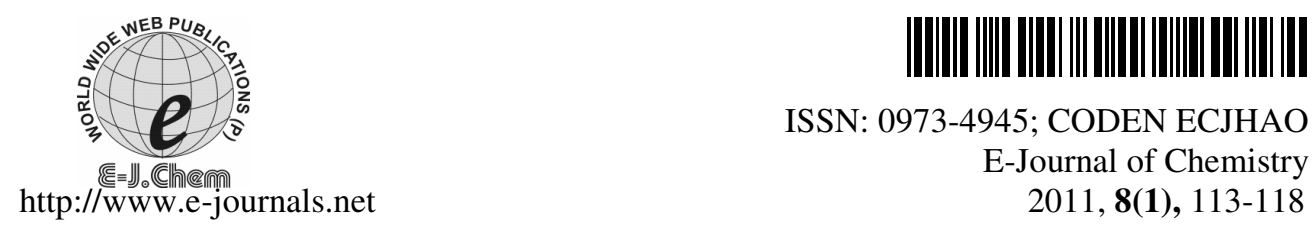

ISSN: 0973-4945; CODEN ECJHAO

E-Journal of Chemistry 2011, 8(1), 113-118

\title{
Synthesis and Biological Evaluation of Coumarinyl Sydnone Derivatives
}

\author{
KESHAV C. PATEL ${ }^{*}$ and HIMANSHU D. PATEL \\ Department of Chemistry \\ Veer Narmad South Gujarat University, Surat-395 007, Gujarat, India \\ drkcpatel55@gmail.com
}

Received 10 May 2010; Accepted 20 July 2010

\begin{abstract}
A novel series of compounds containing coumarinyl sydnone derivatives from 4-methyl-7-hydroxy-8-nitro coumarin were synthesized. The formed compounds have been evaluated by physical methods (melting point, thin layer chromatography, elemental analysis) and by spectral data (IR, ${ }^{1} \mathrm{H}-$ NMR \& ${ }^{13} \mathrm{C}$ NMR). The antimicrobial evaluation of the compounds showed that some of them revealed promising antimicrobial activity.
\end{abstract}

Keywords: Coumarin, Sydnone, Antimicrobial activity, Synthesis.

\section{Introduction}

Sydnone contain a mesoionic aromatic system, which can be depicted with polar resonance structure $^{1}$. Sydnone, the potential basic pharmacodynamic nucleus has been reported to possess a wide variety of biological activities ${ }^{2-4}$ which include antitumor ${ }^{5}$, anticancer ${ }^{6}$, antiinflammatory $^{7}$, antiviral ${ }^{8,9}$, scavenging activity $^{10}$, antimalarial ${ }^{11}$, central nervous system stimulant $^{12}$, HIV Protease inhibitor ${ }^{13}$.

Coumarin and its derivatives have proved a wide spectrum of pharmacological activities. Further coumarin derivatives which on attachment with heterocyclic ring, such as thiazole, triazole $^{14}$ sydnone $^{15}$ exhibit promising biological activities. We report herein the synthesis of a series of novel coumarinyl sydnone derivatives. Its derivatives have taken the coumarin framework to a respectable position in the drugs designing. However, very limited work has been focused on the development of coumarinyl sydnone. As on method of preparation, we used a cyclodehydration of $\alpha$-amino acids $N$-nitroso derivatives, the method which remains the sole useful synthetic rout for the preparation of sydnone since Earl and Mackey's synthesis of sydnone $^{16}$. Review ${ }^{17}$ for the synthesis of 8 -amino coumarin by Pechmann condensation of resorcinol with ethylacetoacetate in presence of condensing agent i.e. $\mathrm{H}_{2} \mathrm{SO}_{4}$ is well known.

\section{Experimental}

The melting points were determined in open capillaries and are uncorrected. The purity of the compounds was controlled by TLC. The spectral data of all the newly synthesized 
compounds, given below, were in full agreement with the proposed structures. Elemental analyses were recorded on Carlo-erba and Perkin Elmer for CHN elements. IR ( $\mathrm{KBr}$ ) spectra were recorded on RZX (Perkin-Elmer) FT-IR model-377 spectrophotometer. ${ }^{1} \mathrm{H}$ NMR spectra were recorded on FT-NMR Cryo-magnet Spectrometer $400 \mathrm{MHz}$ (Bruker) in DMSO-d6 as solvent using TMS as internal reference and chemical shifts $(d)$ are expressed in ppm. ${ }^{13} \mathrm{C}$ NMR spectra were recorded on Varian Mercury VX 300 NMR using DMSO- $d 6$ as solvent and TMS as an internal standard. Mass spectra were recorded on LC-MS Q-ToF micro analyzer (Shimadzu). The synthesis of 8amino-7-(alkoxyl / aryloxyl)-4-methyl-coumarinyl]sydnone is shown in Scheme 1.<smiles>Cc1cc(=O)oc2cc(O)ccc12</smiles>

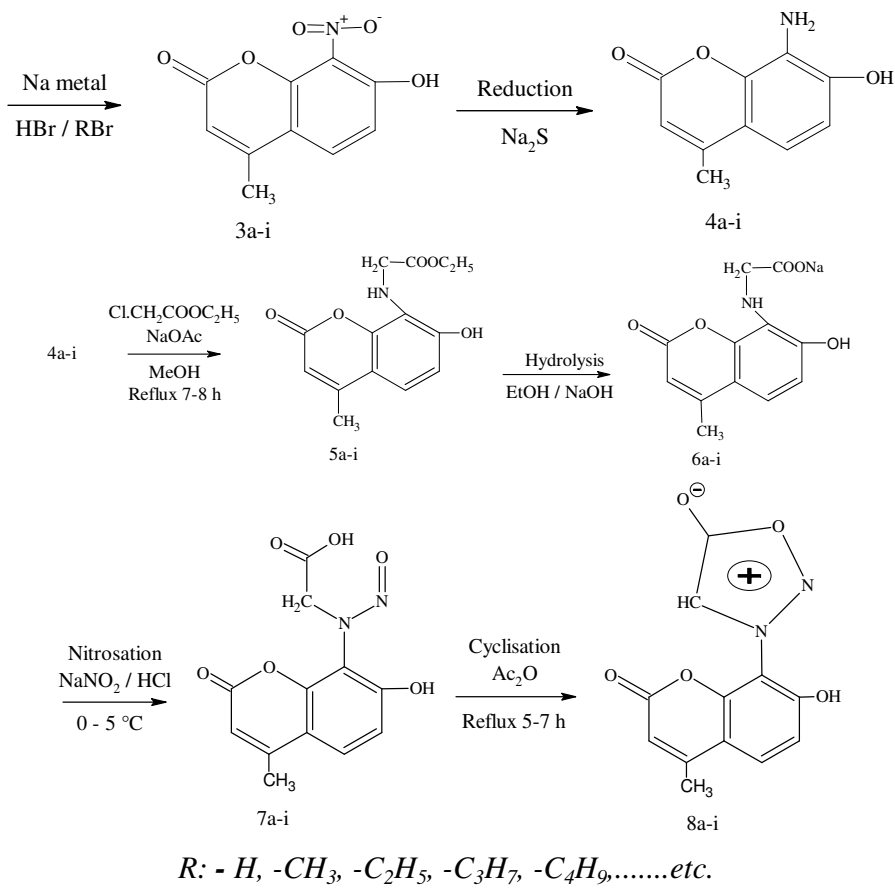

Scheme 1. Synthesis of synthesis of 8-amino-7-(alkoxyl / aryloxyl)-4-methyl-coumarinyl] sydnone. (8a:3-[8-\{7-(hydroxy)-4-methyl\}coumarinyl]sydnone, 8b:3-[8-\{7-(methoxy) -4-methyl\} coumarinyl]sydnone, 8f:3-[8-\{7-(benzyloxy)-4-methyl\} coumarinyl] sydnone)

\section{Synthesis of 4-methyl-7-hydroxy-8-nitro coumarin (2)}

A solution of conc. nitric acid $(4.90 \mathrm{~mL}, 0.0775 \mathrm{~mol})$ in concentrated sulphuric acid $(5.1 \mathrm{~mL})$ was added to a stirred solution of 4-methyl-7-hydroxy coumarin $(13.64 \mathrm{~g}, 0.0775 \mathrm{~mol})$ in conc. sulphuric acid $(30 \mathrm{~mL})$ at such a rate as to keep the temperature below $5{ }^{\circ} \mathrm{C}$. After warming to $20^{\circ} \mathrm{C}$, the reaction mixture was poured into a stirred mixture of ice cold water. The yellow solid that separated was filtered and thoroughly washed with water. The product was recrystalized first from glacial acetic acid and then methyl ethyl ketone to give pure (2). Yield $93 \%$, mp. $256{ }^{\circ} \mathrm{C}$. 


\section{General method for the preparation of alkyl / aryl halide (3a-g)}

A $250 \mathrm{~mL}$ two necked round bottom flask was equipped with a dropping funnel and an efficient double surface condenser, the upper end of which was protected with a calcium chloride gourd drying tube. In this flask, $3.32 \mathrm{~g}(0.14 \mathrm{~mol})$ of lustrous metallic sodium, cut into pieces, was added into $40 \mathrm{~mL}$ of absolute ethanol. When all the sodium had dissolved, the solution was cooled and 0.14 mole of alkyl/aryl bromide and $0.1 \mathrm{~mol}(2)$ were added to it and stirred well and refluxed on water bath for 4-5 h in preparation of alkoxy acetanilide and 8-10 $\mathrm{h}$ in preparation aryl alkoxy derivatives. Then $100 \mathrm{~mL}$ of water was poured through the condenser. The flask was cooled and solid was washed and recrystalized from ethanol. The yields were $80-90 \%$.

\section{Preparation of $\mathbf{3 i}$}

$50 \mathrm{ML}$ three necked round bottom flask fitted with a reflux condenser, a dropping funnel and a stirring unit was used, $0.025 \mathrm{~mol}$ of 4-methyl-7-hydroxy-8-nitro coumarin, $0.85 \mathrm{~g}(0.01 \mathrm{~mol})$ of tetra butyl ammonium hydrogen sulphate, $10 \mathrm{~mL}$ of dichloromethane, $50 \% \mathrm{w} / \mathrm{w}$ aqueous sodium hydroxide ( $2.6 \mathrm{~g}$ sodium hydroxide in $2.6 \mathrm{~g}$ of water) added into it and vigorously stirred for half an hour. The flask was cooled in ice cold water bath and $3.8 \mathrm{~g}(0.03 \mathrm{~mol})$ of dimethyl sulphate was added through a funnel for a period of $1 \mathrm{~h}$. The mixture was stirred vigorously at room temperature until TLC analysis revels the reaction is completed (usually about $3 \mathrm{~h}$ ). $1 \mathrm{~mL}$ of conc. ammonia was added and stirred for further half an hour at room temperature to decompose excess dimethyl sulphate. The reaction mixture was poured into water and then added with $20 \mathrm{~mL}$ of dichloromethane, the organic layer was separated and washed with water until the washing are neutral. The product was dried over magnesium sulphate and evaporated the solvent on a rotatory evaporator. The product is obtained; yield $78 \%,(3.40 \mathrm{~g}), \mathrm{m} . \mathrm{p} .260{ }^{\circ} \mathrm{C}$.

General method for the synthesis 8-amino-7-(alkoxyl / aryloxyl)-4-methyl-coumarin (4a-i)

A solution of sodium hydrosulphite $7 \mathrm{~g},(0.04 \mathrm{~mol})$ in water $30 \mathrm{~mL}$ was added rapidly to pure derivative of 7-(substituted-OH)-4-methyl-8-nitro coumarin $2.37 \mathrm{~g},(0.0107 \mathrm{~mol})$ in $36 \%$ $20 \mathrm{~mL}$ of aqueous ammonia. The mixture was warmed spontaneously with stirring, yellow precipitates or solid that separated was filtered and recrystalized from ethanol.

Synthesis of various alkoxyl / aryloxyl glycines ethyl esters (5a-i)

Derivatives of hydroxyl-8-amino coumarin $(0.10 \mathrm{~mol})$, ethylchloroacetate $(0.10 \mathrm{~mol})$ and sodium acetate crystals $(0.10 \mathrm{~mol})$ in ethanol were heated for $5 \mathrm{~h}$. In the case of aryl alkoxy amino derivatives the equimolar mixture was heated with little excess of ethanol. The reaction mixture was poured into water. The separated oily layer was extracted with ether, washed well with water and dried over anhydrous sodium sulphate.

Synthesis of various alkoxyl / aryloxyl coumarinyl glycines (6a-i)

The crude esters were hydrolyzed by refluxing with $5 \%$ excess of sodium hydroxide in ethanol for $30 \mathrm{~min}$. The crude sodium salts of glycine were filtered and directly used for nitrosation.

Synthesis of various alkoxyl / aryloxyl N-coumarinyl-N-nitrosoglycine (7a-i)

Sodium salt of glycine $(0.10 \mathrm{~mol})$, sodium nitrate $(5 \mathrm{~g})$ in $20 \mathrm{~mL}$ of water was taken in $250 \mathrm{~mL}$ beaker and cooled to $0{ }^{\circ} \mathrm{C}$, the nitroso compound was filtered off and washed well with cold water and dried over sodium hydroxide pellets. Yield $60-65 \%$.

\section{General procedure for the synthesis of $(8 \mathrm{8}-\mathrm{i})$}

Compounds 7 (a-i) $(0.010 \mathrm{~mol})$ and acetic anhydride $6.7 \mathrm{~g}(0.050 \mathrm{~mol})$ were heated for $5-7 \mathrm{~h}$. The mixture was poured into alkaline ice water. The crude product was filtered and washed well with water and dried. The product was recrystallized from benzene-petroleum ether. Yield was 55-60\%. 


\section{3-[8-\{7-(Hydroxy)-4-methyl\}coumarinyl] sydnone (8a)}

Yield 56\%; m.p. $107{ }^{\circ} \mathrm{C}$; Anal. Calcd. for $\mathrm{C}_{12} \mathrm{H}_{10} \mathrm{O}_{5} \mathrm{~N}_{2}$ : C, 54.97; H, 3.84; N, 10.68. Found: C, 54.99; H, 3.88; N, 10.70. IR (KBr, cm $\left.{ }^{-1}\right): 3317,3150,1730,1025 .{ }^{1} \mathrm{H}$ NMR (400 MHz, DMSO- $d_{6}$, ppm): 2.32 (s, 3H, $\left.-\mathrm{CH}_{3}\right), 5.23(\mathrm{~s}, 1 \mathrm{H}, \mathrm{OH}), 6.38-7.10(\mathrm{~s}, 3 \mathrm{H}$, Aromatic $-\mathrm{CH}), 8.20(\mathrm{~s}, 1 \mathrm{H},-\mathrm{NH})$. ${ }^{13} \mathrm{C}-\mathrm{NMR}$ (75 MHz, DMSO-d6, $\delta$, ppm): 161.09, 152.07, 140.91, 135.71, 122.21, 116.61, 114.11, 113.09, 112.49, 109.10, 73.60, 19.35. m/z: 264.17 (100.0\%), 265.05 (13.3\%), 266.18 (1.9\%)

\section{3-[8-\{7-(Methoxy)-4-methyl\} coumarinyl] sydnone $(8 \boldsymbol{b})$}

Yield 52\%; m.p. $132{ }^{\circ} \mathrm{C}$; Anal. Calcd. for $\mathrm{C}_{13} \mathrm{H}_{12} \mathrm{O}_{5} \mathrm{~N}_{2}$ : C, 56.52; H, 4.37; N, 10.14. Found: C, 56.55; H, 4.39; N, 10.16. IR $\left(\mathrm{KBr}, \mathrm{cm}^{-1}\right): 3175,2830,1745,1010 .{ }^{1} \mathrm{H}-\mathrm{NMR}(400 \mathrm{MHz}$, DMSO- $\left.d_{6}, \mathrm{ppm}\right): 2.32\left(\mathrm{~s}, 3 \mathrm{H},-\mathrm{CH}_{3}\right), 3.81\left(\mathrm{~s}, 3 \mathrm{H},-\mathrm{OCH}_{3}\right), 4.08(\mathrm{~s}, 1 \mathrm{H},-\mathrm{CH}), 6.25-7.20$, (s, $3 \mathrm{H}$, Aromatic -CH), $8.20(\mathrm{~s}, 1 \mathrm{H},-\mathrm{NH}) .{ }^{13} \mathrm{C}-\mathrm{NMR}$ (75 MHz, DMSO-d6, $\left.\delta, \mathrm{ppm}\right): 161.90$, $152.67,144.83,135.34,121.21,116.30,113.74,112.11,111.32,109.10,73.60,55.7,19.35$. $\mathrm{m} / \mathrm{z}: 278.07$ (100.0\%), 279.17 (15.0\%), 280.17 (1.1\%)

\section{3-[8-\{7-(Ethoxy)-4-methyl\} coumarinyl] sydnone $(8 \mathrm{c})$}

Yield 54\%; m.p. $129^{\circ} \mathrm{C}$; Anal. Calcd. for $\mathrm{C}_{14} \mathrm{H}_{14} \mathrm{O}_{5} \mathrm{~N}_{2}:$ C, 57.92; H, 4.86; N, 9.64. Found: C, 57.90; $\mathrm{H}, 4.84$; N, 9.67. IR $\left(\mathrm{KBr}, \mathrm{cm}^{-1}\right): 3140,2925,1720,1020 .{ }^{1} \mathrm{H}-\mathrm{NMR}\left(400 \mathrm{MHz}\right.$, DMSO- $d_{6}$, ppm): $1.80(\mathrm{t}, 3 \mathrm{H}), 2.20(\mathrm{~s}, 3 \mathrm{H}), 6.75(\mathrm{~s}, 1 \mathrm{H}), 6.08(\mathrm{~s}, 1 \mathrm{H}), 7.20(\mathrm{~s}, 1 \mathrm{H}), 8.25\left(\mathrm{~s}, \mathrm{C}_{5} \mathrm{H}\right) .{ }^{13} \mathrm{C}-\mathrm{NMR}(75 \mathrm{MHz}$, DMSO- $d 6, \delta$, ppm): 160.91, 152.71, 141.32, 134.82, 120.80, 115.82, 113.30, 112.24, 111.71, 109.12, 73.81, 64.54, 19.35, 14.92. $\mathrm{m} / \mathrm{z}: 292.21$ (100.0\%), 293.05 (15.5\%), 294.10 (2.2\%).

\section{3-[8-\{7-(N-Propoxy)-4-methyl\} coumarinyl]sydnone $(8 \mathrm{~d})$}

Yield 54\%; m.p. $140{ }^{\circ} \mathrm{C}$; Anal. Calcd. for $\mathrm{C}_{15} \mathrm{H}_{16} \mathrm{O}_{5} \mathrm{~N}_{2}$ : C, 59.20; H, 5.29; N, 9.20. Found: C, 59.22; H, 5.31; N, 9.23. IR $\left(\mathrm{KBr}, \mathrm{cm}^{-1}\right): 3210,2890,1715,1015 .{ }^{1} \mathrm{H}-\mathrm{NMR}(400 \mathrm{MHz}$, DMSO- $\left.d_{6}, \mathrm{ppm}\right): 1.1(\mathrm{t}, 3 \mathrm{H}), 1.7-2.0(\mathrm{~m}, 2 \mathrm{H}), 2.04(\mathrm{~s}, 3 \mathrm{H}), 4.0(\mathrm{t}, 2 \mathrm{H}), 6.79(\mathrm{~s}, 1 \mathrm{H}), 6.9(\mathrm{~s}$, $1 \mathrm{H}$, Sydnone), $7.12\left(\mathrm{~s}, \mathrm{C}_{6} \mathrm{H}\right), 8.9\left(\mathrm{~s}, \mathrm{C}_{5} \mathrm{H}\right) .{ }^{13} \mathrm{C}-\mathrm{NMR}(75 \mathrm{MHz}, \mathrm{DMSO}-d 6, \delta, \mathrm{ppm}): 160.90$, $152.67,141.83,134.92,121.20,115.72,113.40,112.17,111.41,109.22,76.40,73.78$, 22.64, 19.35, 10.29. m/z: 306.21 (100.0\%), 307.09 (17.0\%), 308.17 (2.3\%).

\section{3-[8-\{7-(N-Butoxy)-4-methyl\} coumarinyl]sydnone (8e)}

Yield 56\%; m.p. $150{ }^{\circ} \mathrm{C}$; Anal. Calcd. for $\mathrm{C}_{16} \mathrm{H}_{18} \mathrm{O}_{5} \mathrm{~N}_{2}$ : C, 60.37; H, 5.69; N, 8.81. Found: C, 60.40; H, 5.67; N, 8.83. IR $\left(\mathrm{KBr}, \mathrm{cm}^{-1}\right): 3170,2900,1740,1035 .{ }^{1} \mathrm{H}-\mathrm{NMR}(400 \mathrm{MHz}$, DMSO- $\left.d_{6}, \mathrm{ppm}\right): 1.1(\mathrm{t}, 3 \mathrm{H}), 1.8-2.0(\mathrm{~m}, 4 \mathrm{H}), 2.03(\mathrm{~s}, 3 \mathrm{H}), 4.02(\mathrm{t}, 2 \mathrm{H}), 6.75\left(\mathrm{~s}, \mathrm{C}_{3} \mathrm{H}\right), 6.95$ $(\mathrm{s}, 1 \mathrm{H}), 7.02\left(\mathrm{~s}, \mathrm{C}_{6} \mathrm{H}\right), 8.18\left(\mathrm{~s}, \mathrm{C}_{5} \mathrm{H}\right) .{ }^{13} \mathrm{C}-\mathrm{NMR}(75 \mathrm{MHz}, \mathrm{DMSO}-d 6, \delta, \mathrm{ppm}): 160.91$, $152.71,141.32,134.82,120.80,115.82,113.30,112.24,111.71,109.12,73.81,67.95$, 31.76, 19.35, 19.01, 14.92. m/z: 320.17 (100.0\%), 321.21 (17.7\%), 322.07 (2.6\%)

\section{3-[8-\{7-(Benzyloxy)-4-methyl\} coumarinyl] sydnone $(8 \mathrm{f})$}

Yield 50\%; m.p. $142{ }^{\circ} \mathrm{C}$; Anal. Calcd. for $\mathrm{C}_{19} \mathrm{H}_{16} \mathrm{O}_{5} \mathrm{~N}_{2}$ : C, 64.76; H, 4.57; N, 7.95. Found: C, 64.77; H, 4.55; N, 7.98. IR $\left(\mathrm{KBr}, \mathrm{cm}^{-1}\right): 3150,2920,1735,1040 \mathrm{~cm}^{-1} .{ }^{1} \mathrm{H}-\mathrm{NMR}(400 \mathrm{MHz}$, DMSO- $\left.d_{6}, \mathrm{ppm}\right): 2.42\left(\mathrm{~s}, 3 \mathrm{H},-\mathrm{CH}_{3}\right), 4.08(\mathrm{~s}, 1 \mathrm{H},-\mathrm{CH}), 6.25-7.40,(\mathrm{~s}, 8 \mathrm{H}$, Aromatic $-\mathrm{CH})$, $8.20(\mathrm{~s}, 1 \mathrm{H},-\mathrm{NH}) .{ }^{13} \mathrm{C}-\mathrm{NMR}$ (75 MHz, DMSO- $\left.d 6, \delta, \mathrm{ppm}\right): 160.91,157.04,153.07,136.7$, 134.07, 128.42, 123.57, 121.03, 119.01, 114.95, 114.62, 112.54, 109.10, 74.10, 19.26. $\mathrm{m} / \mathrm{z}$ : 340.09 (100.0\%), 341.19 (19.8\%), $342.10(3.0 \%)$

\section{3-[8-\{7-((P-Chloro benzyl)oxy)-4-methyl\} coumarinyl]sydnone (8g)}

Yield 57\%; m.p. $140{ }^{\circ} \mathrm{C}$; Anal. Calcd. for $\mathrm{C}_{19} \mathrm{H}_{15} \mathrm{O}_{5} \mathrm{~N}_{2} \mathrm{Cl}$ : C, 59.00; H, 3.89; N, 7.25. Found: C, 59.03; H, 3.92; N, 7.22. IR $\left(\mathrm{KBr}, \mathrm{cm}^{-1}\right): 3145,2935,1710,1020,752 .{ }^{1} \mathrm{H}-\mathrm{NMR}$ (400 MHz, DMSO- $\left.d_{6}, \mathrm{ppm}\right): 2.41$ (s, 3H, $\left.-\mathrm{CH}_{3}\right), 4.14(\mathrm{~s}, 1 \mathrm{H},-\mathrm{CH}), 6.25-7.65$, (s, 7H, Aromatic - 
$\mathrm{CH}), 8.22(\mathrm{~s}, 1 \mathrm{H},-\mathrm{NH}) .{ }^{13} \mathrm{C}-\mathrm{NMR}(75 \mathrm{MHz}, \mathrm{DMSO}-d 6, \delta, \mathrm{ppm}): 160.89,155.01,152.91$, 136.40, 134.12, 129.45, 127.20, 123.87, 118.88, 114.85, 114.53, 112.49, 109.08, 73.65, 19.41 . $\mathrm{m} / \mathrm{z}: 374.16$ (100.0\%), 376.05 (32.0\%), 375.17 (19.8\%), 377.12 (6.6\%), 376.09 (3.0\%)

3-[8-\{7-((P-Methyl benzyl)oxy)-4-methyl\} coumarinyl]sydnone (8h)

Yield 55\%; m.p. $146{ }^{\circ} \mathrm{C}$; Anal. Calcd. for $\mathrm{C}_{20} \mathrm{H}_{18} \mathrm{O}_{5} \mathrm{~N}_{2}$ : C, 65.56; H, 4.95; N, 7.64. Found: $\mathrm{C}$, 65.57; H, 4.97; N, 7.61. IR $\left(\mathrm{KBr}, \mathrm{cm}^{-1}\right): 3150,2880,1725,1015 .{ }^{1} \mathrm{H}-\mathrm{NMR}(400 \mathrm{MHz}$, DMSO- $\left.d_{6}, \mathrm{ppm}\right): 2.34\left(\mathrm{~s}, 6 \mathrm{H},-\mathrm{CH}_{3}\right), 4.08(\mathrm{~s}, 1 \mathrm{H},-\mathrm{CH}), 6.25-7.15,(\mathrm{~s}, 7 \mathrm{H}$, Aromatic $-\mathrm{CH})$, $8.21(\mathrm{~s}, 1 \mathrm{H},-\mathrm{NH}) .{ }^{13} \mathrm{C}-\mathrm{NMR}$ (75 MHz, DMSO-d6, $\left.\delta, \mathrm{ppm}\right): 160.91,154.01,152.81,136.54$, $134.02,131.45,128.15,123.87,117.08,114.97,114.64,112.59,109.48,73.65,21.51$, 19.35. $\mathrm{m} / \mathrm{z}: 354.21(100.0 \%), 355.13(21.3 \%), 356.14(3.1 \%)$

\section{3-[8-\{7-((P-Fluoro benzyl)oxy)-4-methyl\} coumarinyl]sydnone (8i)}

Yield 58\%; m.p. $139{ }^{\circ} \mathrm{C}$; Anal. Calcd. for $\mathrm{C}_{19} \mathrm{H}_{15} \mathrm{O}_{5} \mathrm{~N}_{2} \mathrm{~F}$ : C, 61.62; H, 4.08; N, 7.56. Found: C, 61.60; H, 4.10; N, 7.59. IR $\left(\mathrm{KBr}, \mathrm{cm}^{-1}\right): 3150,2910,1715,1075,1030 .{ }^{1} \mathrm{H}-\mathrm{NMR}$ (DMSO- $\left.d_{6}, \mathrm{ppm}\right): 2.44$ (s, 3H, $\left.-\mathrm{CH}_{3}\right), 4.12(\mathrm{~s}, 1 \mathrm{H},-\mathrm{CH}), 6.25-7.35$, (s, 7H, Aromatic -CH), $8.14(\mathrm{~s}, 1 \mathrm{H},-\mathrm{NH}) .{ }^{13} \mathrm{C}-\mathrm{NMR}(75 \mathrm{MHz}$, DMSO- $d 6, \delta$, ppm): 160.78, 156.23, 152.71, 136.64, $134.01,123.77,120.09,115.24,114.54,114.92,112.32,109.21,73.75,19.35 . \mathrm{m} / \mathrm{z}: 358.11$ (100.0\%), 359.15 (19.8\%), $360.08(3.0 \%)$

\section{Results and Discussion}

Antimicrobial activity

The synthesized compounds 8(a-i) were tested for their antibacterial activity in vitro in comparison with ampicillin and penicillin as a reference drug using the standard agar disc diffusion method ${ }^{19}$ against bacterial species: Escherichia coli (ATCC 25922), Pseudomonas aeruginosa (ATCC 10145), Staphylococcus aureus (ATCC 25923) and Bacillus subtils (ATCC 6633). All the synthesized compounds were weighed and dissolved in DMSO to prepare exact stock solution of $100 \mu \mathrm{g} / \mathrm{mL}$.

The antimicrobial effects of the substances were tested quantitatively in respective broth media by using double dilution and the zone of inhibition values were determined. The antibacterial were performed in Muller-Hinton broth (MH) (Difco, Detroit, MI) at pH 7.3 and Buffered Yeast Nitrogen Base (Difco, Detroit, MI) at pH 7.0, respectively. Ampicillin (Amp) and penicillin (Pen) were used as standard antibacterial. DMSO with dilution of 1:10 was used as solvent control. The results are shown in Table 1 .

Table 1. Antimicrobial activity of the tested compounds (8a-i)

\begin{tabular}{ccccccccc}
\hline \multirow{2}{*}{$\begin{array}{c}\text { Compd. } \\
\text { No. }\end{array}$} & \multicolumn{2}{c}{$\begin{array}{c}\text { Escherichia } \\
\text { coli }\end{array}$} & \multicolumn{2}{c}{$\begin{array}{c}\text { Staphylococcus } \\
\text { aureus }\end{array}$} & \multicolumn{2}{c}{$\begin{array}{c}\text { Bacillus } \\
\text { subtills }\end{array}$} & \multicolumn{2}{c}{$\begin{array}{c}\text { Pseudomonas } \\
\text { aeruginosa }\end{array}$} \\
\cline { 2 - 9 } & IZ & MIC & IZ & MIC & IZ & MIC & IZ & MIC \\
\hline $\mathbf{8 a}$ & 19 & 250 & 20 & 125 & 23 & 125 & 15 & 500 \\
$\mathbf{8 b}$ & 16 & 250 & 20 & 125 & 25 & 250 & 20 & 125 \\
$\mathbf{8 c}$ & 19 & 125 & 21 & 250 & 21 & 500 & 21 & 250 \\
$\mathbf{8 d}$ & 20 & 500 & 20 & 500 & 26 & 500 & 20 & 250 \\
$\mathbf{8 e}$ & 24 & 125 & 22 & 250 & 20 & 125 & 23 & 500 \\
$\mathbf{8 f}$ & 25 & 250 & 20 & 125 & 21 & 250 & 24 & 125 \\
$\mathbf{8 g}$ & 21 & 500 & 23 & 500 & 20 & 125 & 20 & 500 \\
$\mathbf{8 h}$ & 26 & 125 & 21 & 250 & 19 & 250 & 21 & 500 \\
$\mathbf{8 i}$ & 20 & 500 & 20 & 125 & 19 & 500 & 20 & 125 \\
Amp. & 29 & 125 & 25 & 125 & 28 & 250 & 23 & 125 \\
Pen. & 25 & 125 & 24 & 250 & 27 & 125 & 24 & 125 \\
\hline
\end{tabular}

IZ - Zone of Inhibition in mm., MIC-Minimal Inhibitory Concentration in $\mu \mathrm{g} / \mathrm{mL}$ 
We report the synthesis of some new 8-heterocyclic coumarin derivatives. Novel compounds were synthesized by using resorcinol as a starting material and prepared $\mathbf{1}$, which on nitration gives compound $\mathbf{2}$, then it was reacted with $\mathrm{Na}$ metal \& various alkyl, aryl halide or acetyl derivatives to give compound $\mathbf{3}$ which on nitration, reduction, nitrosation, cyclization etc. we got coumarinyl sydnone ${ }^{18}$. The synthesis of 8-amino-7(alkoxyl / aryloxyl)-4-methyl-coumarin (4a-i) (Scheme 1) was investigated, which on further react with EAA and $\mathrm{NaOAc}$ followed by hydrolysis, nitrosation and cyclisation gives desired sydnone derivatives (8a-i).

\section{Conclusion}

We have synthesized new coumarinyl based sydnone derivatives by conventional method and screened for their antimicrobial activity. The newly synthesized sydnone drugs were successfully characterized by IR, NMR and elemental analysis. From the antimicrobial activity data compound $\mathbf{8 b}, \mathbf{8 g}$ and $\mathbf{8 h}$ showed broad spectrum activity against selected bacteria with respect to standard drug ampicillin and penicilin, other compound showed moderate to weak activity.

\section{Acknowledgment}

The authors thank to Professor and Head, Department of Chemistry, Veer Narmad South Gujarat University, Surat for providing laboratory facilities. The authors express their thanks and gratefully acknowledges the financial support (Fellowship) rendered by UGC-SAP, New Delhi. The authors also thank to SAIF, Chandigarh for IR and NMR spectral analysis. Author sincerely thankful to Mr. Ramprasad Kahar for his kind support during the synthesis work.

\section{References}

1. Mullur S G and Badami B V, Farmaco, 2000, 55(1), 65-67.

2. McNaught A D in, Adv Heterocyclic Chem, Katritzky A R and Boulton A J (Eds.), Advances Heterocyclic Chemistry, Academic Press New York, 1976, 175-319

3. Moustafa M A, Nasr M N, Gineinah M M and Bayoumi W A, Arch Der Pharmazie, 2004, 337, 164-170.

4. Kavali R Jyoti and Badami B V, Farmaco, 2000, 55(5), 406-409.

5. Dunkley C S and Thoman C J, Bioorg Med Chem Lett., 2003, 13(17), 2899-2901.

6. Thamotharan S, Parthasarathi V, Mallur S, Kamble R, Badami B and Linden A, Acta Cryst., 2004, E60, 701-702.

7. Kalluraya B, Rahiman A M, Polish J Chem., 1997, 71, 1049-1052.

8. Pandey V K and Tandon M, Indian J Heterocycl Chem,, 2006, 15, 399-400.

9. Bourinbaiar A S, Tan X and Nagorny R, Acta Virol., 1993, 37, 241-250.

10. Shih M H and Ke F Y, Bioorg Med Chem., 2004, 12, 4633-4633.

11. Friedman M D, Stotter P L, Porter T H and Folkers K, J Med Chem., 1973, 16(11), 1314-1316.

12. Stein, P. Reinhardt, US Patent, 1981, 4289885.

13. Tummino P J, Ferguson D and Hupe D, Biochem Biophy Res Comm., 1994, 201(1), 290-294.

14. Shinge P, Latthe P and Badami B, Synth Commun., 2005, 35(16), 2169-2176.

15. Earl J C and Mackney A W, J Chem Soc., 1935, 899.

16. Sethna S and Phadke R, Org React., 1953, 7, 1-58.

17. Ohta M and. Kato H, Non-Benzenoid Aromatics, 1969, 1, 117-248.

18. Eade R A and Earl J C, J Chem Soc., 1946, 591-593.

19. Arthington-Skaggs B A, Motley M, Warnock D W and Morrison C J, J Clin Microbiol., 2000, 38, 2254-2260. 


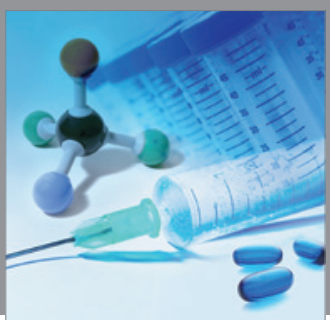

International Journal of

Medicinal Chemistry

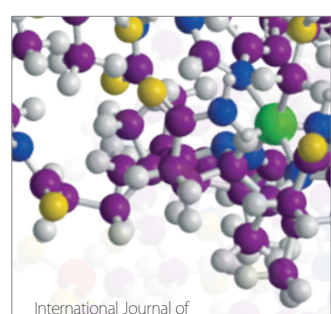

Carbohydrate Chemistry

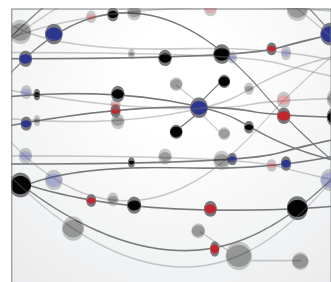

The Scientific World Journal
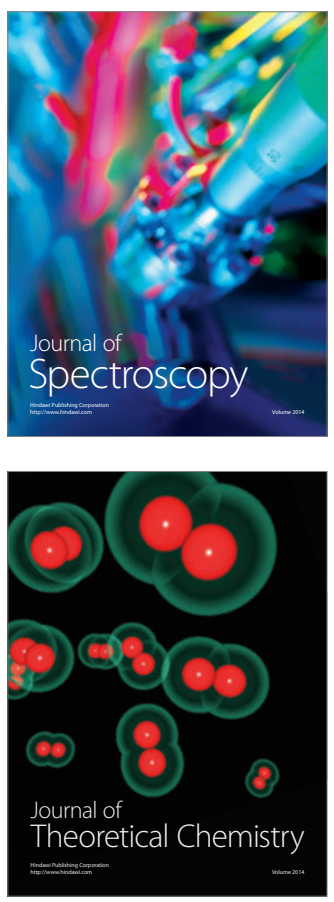
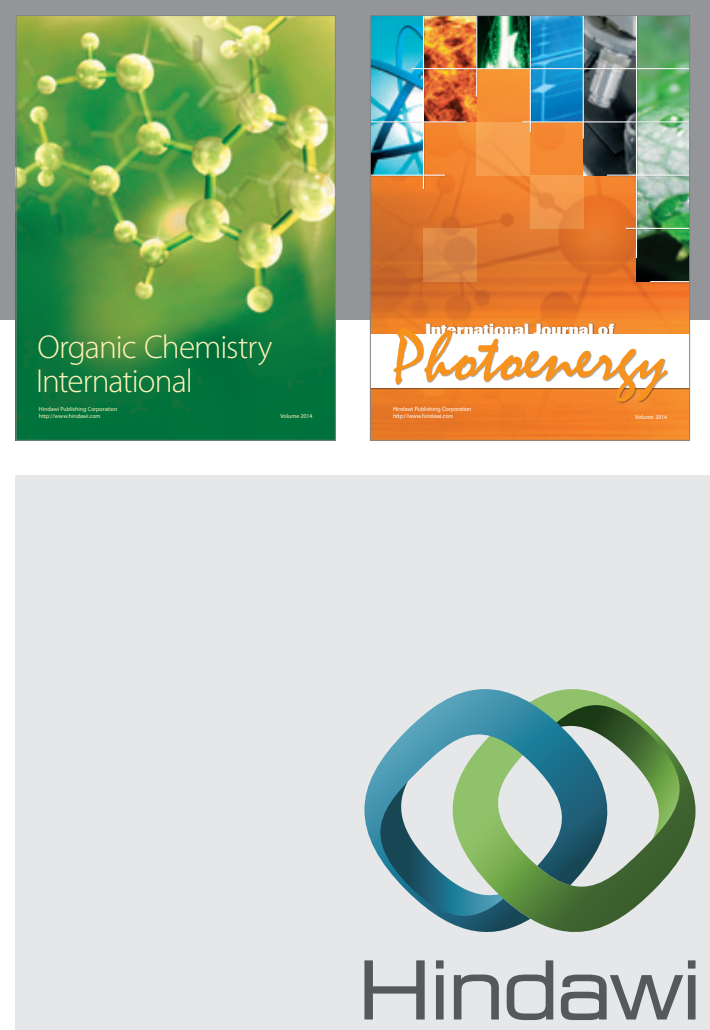

Submit your manuscripts at

http://www.hindawi.com
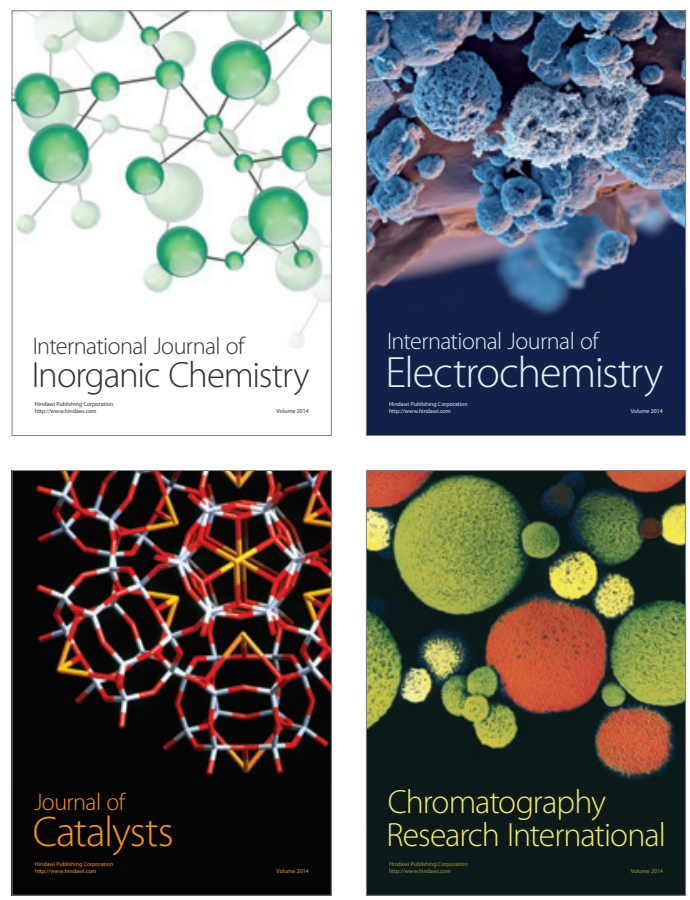
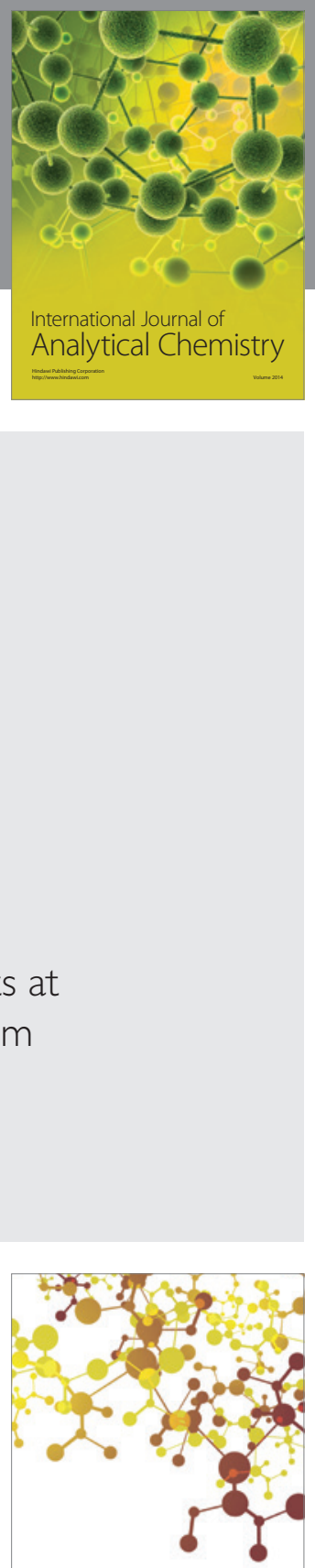

Journal of

Applied Chemistry
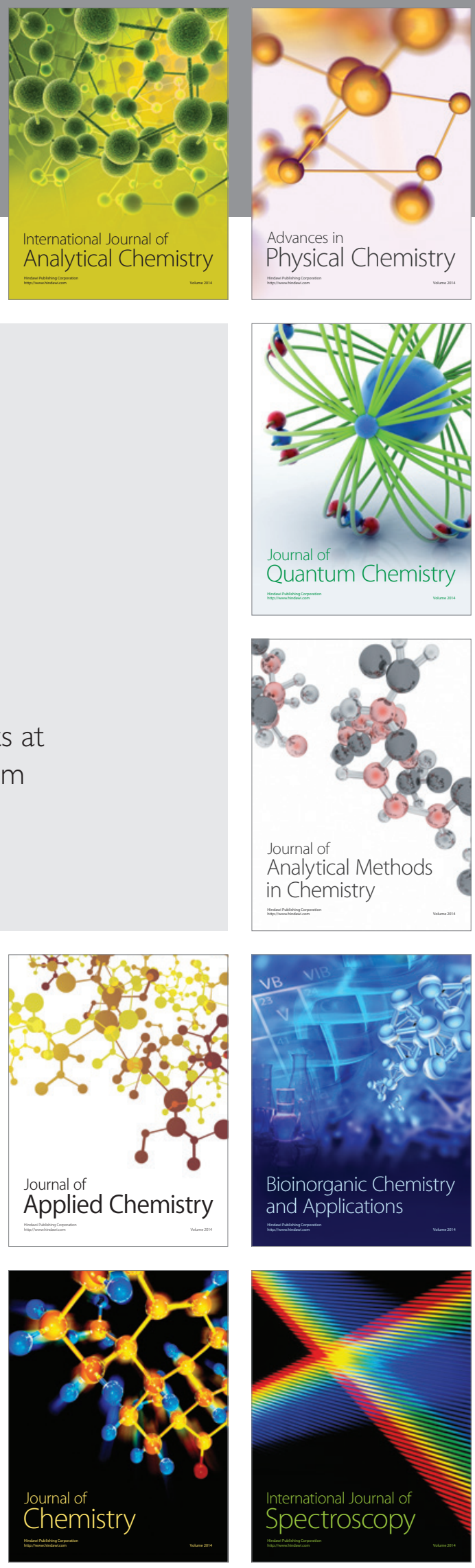